PROCEEDINGS OF THE

AMERICAN MATHEMATICAL SOCIETY

Volume 133, Number 1, Pages 51-56

S 0002-9939(04)07539-2

Article electronically published on August 20, 2004

\title{
THE VALENTINER GROUP AS GALOIS GROUP
}

\author{
TERESA CRESPO AND ZBIGNIEW HAJTO
}

(Communicated by Lance W. Small)

\begin{abstract}
We obtain the complete set of solutions to the Galois embedding problem given by the Valentiner group as a triple cover of the alternating group $A_{6}$.
\end{abstract}

The Valentiner group is a primitive subgroup of the special linear group of order 3 over a field $k$ of characteristic 0 containing the roots of unity of order 15 . It can also be seen as the unique nontrivial triple cover of the alternating group $A_{6}$ in 6 letters. In this paper, we consider the Galois embedding problem given by the Valentiner group and a Galois realization $K$ of $A_{6}$ over a field $k$ containing $\mathbb{Q}\left(\mu_{15}\right)$. We give a correspondence between the solutions of this embedding problem and the $k$-defined points of a certain algebraic variety and obtain all possible solutions to this embedding problem. This is achieved by determining all possible dimension 10 irreducible $k\left[A_{6}\right]$-submodules of $K$ and characterizing among them the ones that are a symmetric cube. In the case when the field $k$ is a differential field we determine the homogeneous linear differential equations of order 3 with Galois group the Valentiner group and Picard-Vessiot extension containing $K$.

Let $F$ denote a field containing $\mathbb{Q}\left(\mu_{15}\right)$. The Valentiner group is the subgroup of the special linear group $\mathrm{SL}(3, F)$ generated by the matrices

$$
\begin{aligned}
& E_{1}=\left(\begin{array}{ccc}
1 & 0 & 0 \\
0 & \zeta^{4} & 0 \\
0 & 0 & \zeta
\end{array}\right) \quad, \quad E_{2}=\left(\begin{array}{ccc}
-1 & 0 & 0 \\
0 & 0 & -1 \\
0 & -1 & 0
\end{array}\right), \\
& E_{3}=\frac{1}{\sqrt{5}}\left(\begin{array}{lll}
1 & 2 & 2 \\
1 & s & t \\
1 & t & s
\end{array}\right), E_{4}=\frac{1}{\sqrt{5}}\left(\begin{array}{ccc}
1 & 2 \lambda_{2} & 2 \lambda_{2} \\
\lambda_{1} & s & t \\
\lambda_{1} & t & s
\end{array}\right),
\end{aligned}
$$

where $\zeta$ is a primitive 5 th root of unity, $s=\zeta^{2}+\zeta^{3}, t=\zeta+\zeta^{4}, \sqrt{5}=t-s$, $\lambda_{1}=\frac{-1 \pm \sqrt{-15}}{4}, \lambda_{2}=\frac{-1 \mp \sqrt{-15}}{4}$. Under the projection of $\operatorname{SL}(3, F)$ onto the projective group $\operatorname{PGL}(3, F)$, the Valentiner group is mapped onto the alternating group $A_{6}$ in 6 letters. The projection of the Valentiner group onto $A_{6}$ can be obtained by mapping the matrices $E_{1}, E_{2}, E_{3}, E_{4}$ to the permutations (12345), (14)(23), (12)(34),(14)(56) of $A_{6}$ respectively (cf. [6]). The Valentiner

Received by the editors June 3, 2003 and, in revised form, October 9, 2003.

2000 Mathematics Subject Classification. Primary 12F12; Secondary 11F80, $12 \mathrm{H} 05$.

The first author was supported in part by BFM2000-0794-C02-01, Spanish Ministry of Education. 
group is the unique nontrivial triple cover of the alternating group $A_{6}$. In the sequel we shall denote the Valentiner group by $3 A_{6}$. By a result of Mestre [5], it is known that the group $3 A_{6}$ appears as the Galois group of $\mathbb{Q}$-regular extensions of $\mathbb{Q}(t)$. More precisely, Mestre gives an explicit construction of $\mathbb{Q}$-regular extensions of $\mathbb{Q}(t)$ with Galois group $A_{6}$ that are embeddable in a $3 A_{6}$-extension. However, no explicit construction of a $3 A_{6}$-extension is known. In this work, we shall give an explicit construction of $3 A_{6}$-extensions valid over a field of characteristic zero containing the roots of unity of order 15 . The main tool for our construction is representation theory, and we are using a generalization of the method given in [2, 3.

We shall consider the faithful representation $\widetilde{\rho}$ of dimension 3 of the group $3 A_{6}$ given by its presentation as a subgroup of $\mathrm{SL}(3, F)$ and the third symmetric power $\rho=\widetilde{\rho}^{(3)}$ of $\widetilde{\rho}$. We recall that $A_{6}$ is the image of $3 A_{6}$ under the projection of $\operatorname{SL}(3, F)$ onto the projective group $\operatorname{PGL}(3, F)$ and that the kernel of $\mathrm{SL}(3, F) \rightarrow \operatorname{PGL}(3, F)$ is the subgroup of the homotheties of ratio a third root of unity. This implies that the representation $\rho$ factors through $A_{6}$. By looking at the character table of the group $A_{6}$ (for example in [4], p. 289), we see that $\rho$ corresponds to the unique irreducible representation of dimension 10 of the group $A_{6}$. The character of this representation is given by:

\begin{tabular}{ccccccc}
1 & $(123)$ & $(12)(34)$ & $(12345)$ & $(13452)$ & $(123)(456)$ & $(1234)(56)$ \\
\hline 10 & 1 & -2 & 0 & 0 & 1 & 0
\end{tabular}

In the case when $k$ is a differential field (of characteristic 0 with algebraically closed field of constants), we shall use the connection between differential equations and group representations. We recall in particular the notion of symmetric power of a homogeneous linear differential equation (see [7, 8]).

Definition 1. Let $L(y)=0$ be a homogeneous linear differential equation of order $n$ over the differential field $k$. Let $\left\{y_{1}, \ldots, y_{n}\right\}$ be a fundamental set of solutions of $L(y)=0$. We call the symmetric power of order $m$ of $L(y)=0$ the differential equation $L^{(m)}(y)=0$ whose solution space is spanned by $\left\{y_{1}^{i_{1}} \ldots y_{n}^{i_{n}} \mid i_{1}+\cdots+i_{n}=\right.$ $m\}$.

Let $k$ be a differential field of characteristic 0 with algebraically closed field of constants $\mathcal{C}$ and $L(y)=0$ a homogeneous linear irreducible differential equation of order 3 over $k$ with Galois group $3 A_{6}$. Then the third symmetric power $L^{(3)}(y)=0$ of $L(y)=0$ is an irreducible linear differential equation of order 10 with Galois group $A_{6}$, and the representation of $A_{6}$ obtained from the action of $A_{6}$ on the solution space is the representation $\rho$ above.

We shall use the following lemma on representations.

Lemma 1. Let $V$ be a k-vector space of dimension $n$ and $\varphi: G \rightarrow \mathrm{GL}(V)$ an absolutely irreducible representation. We consider:

$$
\varphi^{m}=\overbrace{\varphi \oplus \cdots \oplus \varphi}^{m}: G \rightarrow \mathrm{GL}\left(V^{m}\right)
$$

where $V^{m}=\overbrace{V \oplus \cdots \oplus V}^{m}$, and we fix monomorphisms $f_{j}: V \rightarrow V^{m}$ such that $\pi_{j} \circ f_{j}: V \rightarrow V$, where $\pi_{j}$ is the projection on the $j$-component, is an isomorphism of $G$-modules, $1 \leq j \leq m$. 
Then every invariant $k$-subspace of $V^{m}$ isomorphic to $V$ as a $G$-module is of the form

$$
\left\langle\left(\sum_{j} a_{j} f_{j}\left(v_{i}\right)\right)_{1 \leq i \leq n}\right\rangle
$$

for some $\left(a_{1}, \ldots, a_{m}\right) \in k^{m} \backslash\{(0, \ldots, 0)\}$ and $\left(v_{1}, \ldots, v_{n}\right)$ a $k$-basis of $V$.

Proof. Let $W$ be an invariant $k$-subspace of $V^{m}, G$-isomorphic to V. The thesis of the lemma is equivalent to the composition

$$
V \simeq W \hookrightarrow V \oplus \cdots \oplus V \stackrel{\pi_{j}}{\longrightarrow} V
$$

being a homothety for each $j$.

This composition is a $G$-endomorphism of $V$ and, since $\varphi$ is irreducible, either 0 or a G-automorphism of $V$. Now, using again the irreducibility of $\varphi$, we obtain that each $G$-automorphism of $V$ is a homothety.

We state now our main result.

Theorem 1. Let $k$ be a field of characteristic 0 , containing the 15 th roots of unity. Let $P(X) \in k[X]$ be a polynomial of degree 6 with Galois group $A_{6}, K$ a splitting field of the polynomial $P(X)$.

There exists an algebraic variety $Q$ in the dimension 9 projective space defined over $k$, such that the Galois embedding problem

$$
3 A_{6} \rightarrow A_{6} \simeq \operatorname{Gal}(K \mid k)
$$

is solvable if and only if $Q$ has a point defined over $k$.

Let $V_{j}, 1 \leq j \leq 10$, be ten $k$-vector subspaces of $K$ such that the action of $A_{6}$ on each of them corresponds to the unique irreducible dimension 10 representation $\rho$ of $A_{6}$ and such that the sum of the $V_{j}$ is a direct sum. Let $F_{i j}, 1 \leq i \leq 10$, be a basis of $V_{j}, 1 \leq j \leq 10$, such that $F_{i j} \mapsto F_{i k}$ defines an isomorphism of $A_{6}$-modules from $V_{j}$ onto $V_{k}$. The vectors $F_{i j}$ can be chosen such that:

a) The extension $\widetilde{K}=K\left(\sqrt[3]{G_{1}}\right)$, where $G_{1}=\sum_{j} a_{j} F_{1 j}$, with $\left(a_{1}, \ldots, a_{10}\right)$ in $Q(k)$, is a solution to $(G E P)$.

b) When $k$ is a differential field with algebraically closed field of constants $\mathcal{C}$, then, for $\left(a_{1}, \ldots, a_{10}\right) \in Q(k),\left\{H_{1}, H_{2}, H_{3}\right\}$, where $H_{i}=\sqrt[3]{\sum_{j} a_{j} F_{i j}}, 1 \leq i \leq 3$, is a basis of the solution space of a differential equation of order 3 with Galois group $3 A_{6}$ over $k$.

The elements $F_{i j}$ and the variety $Q$ can be obtained explicitly. From a point in $Q(k)$ we obtain then explicitly the element $G_{1}$ in a) and the differential equation in b) with Galois group $3 A_{6}$.

Proof. Assume (GEP) is solvable and let $\widetilde{K}$ be a solution. We consider $\widetilde{K}$ as a $k$-vector space and the representation

$$
\phi: 3 A_{6} \rightarrow \mathrm{GL}(\widetilde{K})
$$

given by the Galois action. By the normal basis theorem, $\phi$ is the regular representation, and so $\widetilde{K}$ contains an invariant $k$-vector space $U=\left\langle u_{1}, u_{2}, u_{3}\right\rangle$ of dimension 3 such that $\phi_{\mid U}$ is equivalent to the faithful unimodular representation $\widetilde{\rho}$ of $3 A_{6}$. The symmetric cube of the representation $\phi_{\mid U}$ provides a $k$-vector space of dimension 10 contained in $K$, which is an irreducible invariant subspace of the representation of $A_{6}$ on $K$ given by the Galois action. Moreover $\widetilde{K}=K\left(u_{1}\right)$. 
Now let $P(X) \in k[X]$ be a polynomial of degree 6 , with Galois group $A_{6}$, and let $K$ denote a splitting field of $P(X)$. We consider the representation

$$
A_{6} \rightarrow \mathrm{GL}(K)
$$

given by the Galois action. Again by the normal basis theorem, this representation is the regular one and so contains the dimension 10 irreducible representation $\rho=$ $\widetilde{\rho}^{(3)}$ ten times. We shall determine explicitly ten $k$-subspaces $V_{i}$ of dimension 10 of $K$ such that their sum is direct and such that the Galois action on $V_{i}, i=1, \ldots, 10$, corresponds to $\rho$. To this end, we apply the following lemma, proven in [1], to the permutation representation

$$
\sigma: A_{6} \rightarrow \mathcal{S}_{\left\{e_{1}, \ldots, e_{6}\right\}} \rightarrow \mathrm{GL}(6, k) .
$$

Lemma 2. If $\sigma: G \hookrightarrow \mathrm{GL}(V)$ is a faithful permutation representation of a finite group $G$, then any irreducible representation of $G$ is contained in some symmetric power $\sigma^{(N)}$ of $\sigma$.

By computing the characters of the symmetric powers, we obtain that $\rho$ is contained in the third symmetric power of $\sigma$. The corresponding invariant subspace $V$ of dimension 10 is contained in the dimension 15 invariant subspace $\left\langle e_{i j}=e_{i}^{2} e_{j}-e_{i} e_{j}^{2}\right\rangle_{1 \leq i<j \leq 6}$.

The following vectors written in the basis $e_{i j}$ (taken in lexicographic order) form a basis of $V$ :

$$
\begin{aligned}
& v_{1}=(0,1,-1,1,-1,-1,1,-1,1,0,0,0,0,0,0) \\
& v_{2}=(-1,1,0,0,0,0,-1,1,-1,1,-1,1,0,0,0) \\
& v_{3}=(0,-1,1,0,0,1,-1,0,0,0,1,-1,-1,1,0) \\
& v_{4}=(0,0,-1,0,1,0,1,0,-1,-1,0,1,-1,0,-1) \\
& v_{5}=(0,0,1,0,-1,0,-1,0,1,0,0,0,0,0,0) \\
& v_{6}=(0,1,0,0,-1,-1,0,0,1,0,0,0,0,0,0) \\
& v_{7}=(0,0,0,0,0,0,-1,0,1,1,0,-1,0,0,0) \\
& v_{8}=(0,0,0,0,0,0,0,1,-1,0,-1,1,0,0,0) \\
& v_{9}=(0,0,0,0,0,1,0,-1,0,1,0,0,1,0,0) \\
& v_{10}=(0,0,0,0,0,0,0,0,0,-1,1,0,-1,0,0) .
\end{aligned}
$$

The restriction to $V$ of the $k$-morphisms

$$
e_{i j} \mapsto x_{i}^{m} x_{j}^{n}-x_{i}^{n} x_{j}^{m}
$$

for $1 \leq n<m \leq 5$, where $x_{1}, \ldots, x_{6}$ are the roots of the polynomial $P(X)$ in $K$, are monomorphisms, which we denote by $f_{j}, 1 \leq j \leq 10$, and their images are ten subspaces of dimension 10, isomorphic as $A_{6}$-modules to $V$ and such that their sum is direct. In order to check this last statement, by using Lemma 1, it is enough to check that the ten vectors $f_{j}\left(v_{i}\right)$, for a fixed $i$, are linearly independent.

By Lemma 1, every invariant subspace of $K$ isomorphic to $V$ as an $A_{6}$-module is of the form

$$
\left\langle\left(\sum_{j} a_{j} f_{j}\left(v_{i}\right)\right)_{1 \leq i \leq 10}\right\rangle
$$

for some $\left(a_{1}, \ldots, a_{10}\right) \in \mathbb{P}_{9}(k)$.

Now let $\left(u_{1}, u_{2}, u_{3}\right)$ be a basis in which the representation $\widetilde{\rho}$ is given by the matrices $E_{1}, E_{2}, E_{3}, E_{4}$ above. We take in $V$ a basis $F_{1}, \ldots, F_{10}$ such that assigning the vectors in the basis

$$
\left(u_{1}^{3}, u_{2}^{3}, u_{3}^{3}, u_{1}^{2} u_{2}, u_{1} u_{2}^{2}, u_{1}^{2} u_{3}, u_{1} u_{3}^{2}, u_{2}^{2} u_{3}, u_{2} u_{3}^{2}, u_{1} u_{2} u_{3}\right)
$$

of the third symmetric power $U^{(3)}$ of the $3 A_{6}$-module $U=\left\langle u_{1}, u_{2}, u_{3}\right\rangle$ to the vectors 
$F_{i}$ is a morphism of $A_{6}$-modules. By computation, we obtain that the basis change matrix from the basis $\left(F_{i}\right)_{1 \leq i \leq 10}$ to the basis $\left(v_{i}\right)_{1 \leq i \leq 10}$ is:

$$
C=\frac{1}{8}\left(C_{1}+\sqrt{-15} C_{2}\right)
$$

where $C_{1}$ and $C_{2}$ are the following matrices written down by rows:

$C_{1}=\left[\left[15\left(3 \zeta^{2}-\zeta+3\right), 120(-3 s+1), 120\left(3 s+\zeta^{2}+3 \zeta\right), 10\left(17 s+26 \zeta^{2}+43 \zeta+\right.\right.\right.$ $17), 20\left(-11 s-7 \zeta^{2}-11 \zeta\right), 10\left(-17 s+17 \zeta^{2}+26 \zeta+26\right), 20(11 s-7), 40\left(7 s+16 \zeta^{2}+\right.$ $\left.23 \zeta+7), 40\left(-7 s+7 \zeta^{2}+16 \zeta+16\right), 20\left(9 \zeta^{2}+12 \zeta+9\right)\right],\left[15\left(3 \zeta^{2}-\zeta+3\right), 120\left(s-z^{2}-\right.\right.$ $3 \zeta-3), 120\left(-s-3 \zeta^{2}-4 \zeta-1\right), 10(26 s-17), 20\left(7 s+11 \zeta^{2}+18 \zeta+7\right),-10\left(26 s+17 \zeta^{2}+\right.$ $26 \zeta), 20\left(-7 s+7 \zeta^{2}+11 \zeta+11\right), 40(16 s-7),-40\left(16 s+7 \zeta^{2}+16 \zeta\right), 20\left(9 \zeta^{2}+12 \zeta+\right.$ 9)], $\left[0,240\left(4 s+4 \zeta^{2}+7 \zeta+1\right), 240\left(-4 s+\zeta^{2}+3 \zeta+4\right), 80\left(s+6 \zeta^{2}+8 \zeta+4\right), 80\left(4 s-\zeta^{2}-\right.\right.$ $3 \zeta-4), 80\left(-s+4 \zeta^{2}+7 \zeta+6\right),-80\left(4 s+4 \zeta^{2}+7 \zeta+1\right),-80\left(s+6 \zeta^{2}+8 \zeta+4\right), 80\left(s-4 \zeta^{2}-\right.$ $7 \zeta-6), 0],\left[0,240\left(6 s+\zeta^{2}+3 \zeta-1\right), 240\left(-6 s-\zeta^{2}-3 \zeta+1\right), 80\left(-6 s-\zeta^{2}-3 \zeta+1\right), 80(6 s+\right.$ $\left.\zeta^{2}+3 \zeta-1\right), 80\left(6 s+\zeta^{2}+3 \zeta-1\right), 80\left(-6 s-\zeta^{2}-3 \zeta+1\right), 80\left(6 s+\zeta^{2}+3 \zeta-1\right), 80\left(-6 s-\zeta^{2}-\right.$ $3 \zeta+1), 0],\left[30\left(16 \zeta^{2}+23 \zeta+16\right), 120\left(s-2 \zeta^{2}-\zeta+1\right), 120\left(-s+\zeta^{2}-2 \zeta-2\right),-10(39 s+\right.$ $\left.23 \zeta^{2}+39 \zeta\right), 20\left(-3 s+3 \zeta^{2}+14 \zeta+14\right), 10(39 s-23), 20\left(3 s+14 \zeta^{2}+17 \zeta+3\right), 40(21 s+$ $\left.\left.17 \zeta^{2}+21 \zeta\right), 40(-21 s+17), 20\left(21 \zeta^{2}+33 \zeta+21\right)\right],\left[-25\left(21 \zeta^{2}+27 \zeta+21\right), 120(6 s+\right.$ $\left.6 \zeta^{2}+13 \zeta+4\right), 120\left(-6 s+4 \zeta^{2}+7 \zeta+6\right), 10\left(-34 s+21 \zeta^{2}+28 \zeta+39\right), 20\left(22 s-8 \zeta^{2}-\right.$ $19 \zeta-22), 10\left(34 s+39 \zeta^{2}+62 \zeta+21\right),-20\left(22 s+22 \zeta^{2}+41 \zeta+8\right),-40\left(14 s+39 \zeta^{2}+\right.$ $\left.52 \zeta+21), 40\left(14 s-21 \zeta^{2}-38 \zeta-39\right),-300\left(2 \zeta^{2}+3 \zeta+2\right)\right],\left[15\left(-3 \zeta^{2}+\zeta-3\right), 120(-5 s-\right.$ $\left.3 \zeta^{2}-4 \zeta+2\right), 120\left(5 s+2 \zeta^{2}+\zeta-3\right), 10\left(-25 s-14 \zeta^{2}-12 \zeta+11\right),-20\left(5 s+14 \zeta^{2}+17 \zeta+\right.$ 9), $10\left(25 s+11 \zeta^{2}+13 \zeta-14\right), 20\left(5 s-9 \zeta^{2}-12 \zeta-14\right), 40\left(-5 s+26 \zeta^{2}+48 \zeta+31\right), 40(5 s+$ $\left.31 \zeta^{2}+53 \zeta+26\right),-60\left(3 \zeta^{2}+4 \zeta+3\right), 30\left(-3 \zeta^{2}+\zeta-3\right), 40\left(-36 s-24 \zeta^{2}-27 \zeta+3\right), 40(36 s+$ $\left.3 \zeta^{2}+9 \zeta-24\right), 10\left(-27 s+50 \zeta^{2}+100 \zeta+84\right),-20\left(24 s+17 \zeta^{2}+31 \zeta+4\right), 10\left(27 s+84 \zeta^{2}+\right.$ $127 \zeta+50), 20\left(24 s-4 \zeta^{2}-7 \zeta-17\right), 40\left(-27 s+10 \zeta^{2}+20 \zeta+24\right), 40\left(27 s+24 \zeta^{2}+47 \zeta+\right.$ $\left.10),-120\left(3 \zeta^{2}+4 \zeta+3\right)\right],\left[-25\left(21 \zeta^{2}+27 \zeta+21\right),-600\left(s+\zeta^{2}+\zeta\right), 600(s-1), 250(-s+\right.$ $\left.\zeta^{2}+2 \zeta+2\right), 100(-s+1), 250\left(s+2 \zeta^{2}+3 \zeta+1\right), 100\left(s+\zeta^{2}+\zeta\right), 200\left(-s+\zeta^{2}+2 \zeta+\right.$ $\left.2), 200\left(s+2 \zeta^{2}+3 \zeta+1\right),-300\left(2 \zeta^{2}+3 \zeta+2\right)\right],\left[-30\left(16 \zeta^{2}+23 \zeta+16\right),-240\left(11 s+7 \zeta^{2}+\right.\right.$ $11 s), 240(11 s-7), 20\left(-s+\zeta^{2}+18 \zeta+18\right), 40(-17 s+9), 20\left(1+18 \zeta^{2}+19 \zeta+1\right), 40(17 s+$ $\left.\left.\left.9 \zeta^{2}+17 \zeta\right), 80\left(-11 s+11 \zeta^{2}+18 \zeta+18\right), 80\left(11 s+18 \zeta^{2}+29 \zeta+11\right),-60\left(7 \zeta^{2}+11 \zeta+7\right)\right]\right] ;$ $C_{2}=\left[\left[15\left(5 \zeta^{2}+9 \zeta+5,120(-s+1), 120\left(s+\zeta^{2}+\zeta\right),-10\left(s+2 \zeta^{2}+3 \zeta+1\right), 60\left(s+\zeta^{2}+\right.\right.\right.\right.$ $\zeta), 10\left(s-\zeta^{2}-2 \zeta-2\right), 60(-s+1),-40\left(s+2 \zeta^{2}+3 \zeta+1\right), 40\left(s-\zeta^{2}-2 \zeta-2\right),-20\left(5 \zeta^{2}+\right.$ $8 \zeta+5)],\left[15\left(5 \zeta^{2}+9 \zeta+5\right), 120\left(s-\zeta^{2}-\zeta-1\right),-120\left(s+\zeta^{2}+2 \zeta+1\right), 10(-2 s+\right.$ $1),-60\left(s+\zeta^{2}+2 \zeta+1\right), 10\left(2 s+\zeta^{2}+2 \zeta\right), 60\left(s-\zeta^{2}-\zeta-1\right), 40(-2 s+1), 40\left(2 s+\zeta^{2}+\right.$ $\left.2 \zeta),-20\left(5 \zeta^{2}+8 \zeta+5\right)\right],[0,0,0,0,0,0,0,0,0,0],[0,0,0,0,0,0,0,0,0,0],[30 \zeta,-120(s+$ $\left.2 \zeta^{2}+3 \zeta+1\right), 120\left(s-\zeta^{2}-2 z-2\right),-10\left(s+\zeta^{2}+\zeta\right), 60\left(s-\zeta^{2}-2 \zeta-2\right), 10(s-1),-60(s+$ $\left.\left.2 \zeta^{2}+3 \zeta+1\right),-40\left(s+\zeta^{2}+\zeta\right), 40(s-1),-20\left(5 \zeta^{2}+9 \zeta+5\right)\right],\left[-15\left(5 \zeta^{2}+11 \zeta+5\right), 120(2 s+\right.$ $\left.2 \zeta^{2}+3 \zeta\right), 120(-2 s+\zeta+2), 10\left(2 s+3 \zeta^{2}+4 \zeta+1\right), 60(-2 s+\zeta+2), 10\left(-2 s+\zeta^{2}+2 \zeta+\right.$ $3), 60\left(2 s+2 \zeta^{2}+3 \zeta\right), 40\left(2 s+3 \zeta^{2}+4 \zeta+1\right), 40\left(-2 s+\zeta^{2}+2 \zeta+3\right), 20\left(10 \zeta^{2}+17 \zeta+\right.$ $10)],\left[-15\left(5 \zeta^{2}+9 \zeta+5\right), 120\left(s+3 \zeta^{2}+4 \zeta+2\right), 120\left(-s+2 \zeta^{2}+3 \zeta+3\right), 10\left(s-2 \zeta^{2}-4 \zeta-\right.\right.$ $3), 60\left(-s+2 \zeta^{2}+3 \zeta+3\right),-10\left(s+3 \zeta^{2}+5 \zeta+2\right), 60\left(s+3 \zeta^{2}+4 \zeta+2\right), 40\left(s-2 \zeta^{2}-4 \zeta-\right.$ $3),-40\left(s+3 \zeta^{2}+5 \zeta+2\right), 20\left(5 \zeta^{2}+8 \zeta+5\right),-30\left(5 \zeta^{2}+9 \zeta+5\right), 120\left(4 \zeta^{2}+5 \zeta+3\right), 120\left(3 \zeta^{2}+\right.$ $5 \zeta+4), 10\left(3 s-2 \zeta^{2}-4 \zeta-4\right), 60\left(3 \zeta^{2}+5 \zeta+4\right),-10\left(3 s+4 \zeta^{2}+7 \zeta+2\right), 60\left(4 \zeta^{2}+\right.$ $\left.5 \zeta+3), 40\left(3 s-2 \zeta^{2}-4 \zeta-4\right),-40\left(3 s+4 \zeta^{2}+7 \zeta+2\right), 40\left(5 \zeta^{2}+8 \zeta+5\right)\right],\left[-15\left(5 \zeta^{2}+\right.\right.$ $11 \zeta+5), 120\left(s+\zeta^{2}+\zeta\right), 120(-s+1), 10\left(s-\zeta^{2}-2 \zeta-2\right), 60(-s+1),-10\left(s+2 \zeta^{2}+\right.$ $3 \zeta+1), 60\left(s+\zeta^{2}+\zeta\right), 40\left(s-\zeta^{2}-2 \zeta-2\right),-40\left(s+2 \zeta^{2}+3 \zeta+1\right), 20\left(10 \zeta^{2}+17 \zeta+\right.$

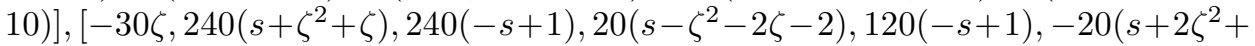
$\left.\left.3 \zeta+1), 120\left(s+\zeta^{2}+\zeta\right), 80\left(s-\zeta^{2}-3 \zeta-3\right),-80\left(s+2 \zeta^{2}+3 \zeta+1\right), 20\left(5 \zeta^{2}+9 \zeta+5\right)\right]\right]$. 
Let $F_{i j}=f_{j}\left(F_{i}\right), 1 \leq i, j \leq 10$. Any invariant subspace of $K$ isomorphic to $V$ as an $A_{6}$-module then has a basis

$$
G_{i}=\sum_{j} a_{j} F_{i j}, 1 \leq i \leq 10,
$$

with $\left(a_{1}, \ldots, a_{10}\right) \in \mathbb{P}_{9}(k)$, which behaves under the $A_{6}$-action as the basis in $U^{(3)}$.

The subspace $\left\langle G_{1}, \ldots, G_{10}\right\rangle$ is a symmetric cube if the elements $G_{i}$ can be written as $\left(u_{1}^{3}, u_{2}^{3}, u_{3}^{3}, u_{1}^{2} u_{2}, u_{1} u_{2}^{2}, u_{1}^{2} u_{3}, u_{1} u_{3}^{2}, u_{2}^{2} u_{3}, u_{2} u_{3}^{2}, u_{1} u_{2} u_{3}\right)$ for some parameters $u_{1}, u_{2}, u_{3}$. This is equivalent to the set of polynomials $\mathcal{P}=\left\{X_{1} X_{2}-X_{4} X_{5}, X_{1} X_{3}-\right.$ $\left.X_{6} X_{7}, X_{2} X_{3}-X_{8} X_{9}, X_{1} X_{10}-X_{4} X_{6}, X_{2} X_{10}-X_{5} X_{8}, X_{1} X_{5}-X_{4}^{2}, X_{5} X_{7}-X_{10}^{2}\right\}$ vanishing in $G_{1}, \ldots, G_{10}$. Since the $F_{i j}$ are known elements in the field $K$, the condition above provides degree 2 homogeneous equations defined over $K$ on the coordinates $\left(a_{1}, \ldots, a_{10}\right)$ and so defines an algebraic variety $Q$ in $\mathbb{P}_{9}(K)$. Now, we can add polynomials to the set $\mathcal{P}$ in order to obtain a family of equations with the property that, by the action of $A_{6}$, each of these equations is transformed into a linear combination of the equations in the family with coefficients in the field $\mathbb{Q}\left(\mu_{15}\right)$, and so we obtain that the variety $Q$ is defined over $k$.

The field $K\left(\sqrt[3]{G_{1}}\right)$ is a cubic extension of $K$ containing the elements $\sqrt[3]{G_{1}}, \sqrt[3]{G_{2}}$, $\sqrt[3]{G_{3}}$ which generate a $k$-subspace on which the action of $3 A_{6}$ corresponds to the representation $\widetilde{\rho}$, and so we obtain the statements a) and b) in the theorem.

\section{ACKNOWLEDGMENT}

We thank Professor Jean-Pierre Serre for his valuable comments on an earlier version of our work.

\section{REFERENCES}

1. T. Crespo, Z. Hajto, Finite linear groups as differential Galois groups, Bull. Pol. Acad. Sci. Math. 49 n.4 (2001), 363-375. MR1872670 (2002k:12016)

2. T. Crespo, Z. Hajto, Recouvrements doubles comme groupes de Galois différentiels, C.R. Acad. Sci. Paris, Série I, 333 (2001), 271-274. MF1854763 (2002k:12017)

3. T. Crespo, Z. Hajto, Differential Galois realization of double covers, Annales de l'Institut Fourier, 52 (2002), 1017-1025. MR 1926670 (2004e:12006)

4. I.M. Isaacs, Character theory of finite groups, Academic Press, 1976. MR0460423 (57:417)

5. J-F. Mestre, Extensions $\mathbb{Q}$-régulières de $\mathbb{Q}(t)$ de groupe de Galois $6 A_{6}$ et $6 A_{7}$, Israel J. Math. 107 (1998), 333-341. MR.1658587 (99i:12003)

6. G.A. Miller, H.F. Blichfeldt, L.E. Dickson, Theory and applications of finite groups, John Wiley and Sons, Inc., 1916.

7. M.F. Singer, An outline of differential Galois theory, in Computer Algebra and Differential Equations, E. Tournier ed., Academic Press, 1989, pp. 3-57. MR1038057 (91a:12011)

8. M.F. Singer, M. van der Put, Galois Theory of Linear Differential Equations, Springer Verlag, Berlin, 2003. MR1960772 (2004c:12010)

Departament d’Àlgebra i Geometria, Universitat de Barcelona, Gran Via de les Corts Catalanes 585, 08007 Barcelona, Spain

E-mail address: crespo@mat.ub.es

Departament D’Àlgebra i Geometria, Universitat de Barcelona, Gran Via de les Corts Catalanes 585, 08007 Barcelona, Spain

Current address: Zakład Matematyki, Akademia Rolnicza, al. Mickiewicza 24/28, 30-059 Kraków, Poland

E-mail address: rmhajto@cyf-kr.edu.pl 